\title{
Minimizing an Electron Flow to Molecular Oxygen in Photosynthetic Electron Transfer Chain: An Evolutionary View
}

\author{
Marina A. Kozuleva*, Boris N. Ivanov, Daria V. Vetoshkina and \\ Maria M. Borisova-Mubarakshina*
}

Institute of Basic Biological Problems (RAS), Pushchino, Russia

OPEN ACCESS

Edited by:

Chikahiro Miyake,

Kobe University, Japan

Reviewed by:

Yoshitaka Nishiyama,

Saitama University, Japan

Christine Helen Foyer,

University of Leeds, United Kingdom

*Correspondence:

Marina A. Kozuleva

kozuleva@gmail.com

Maria M. Borisova-Mubarakshina

mubarakshinamm@gmail.com

Specialty section:

This article was submitted to

Plant Physiology,

a section of the journal

Frontiers in Plant Science

Received: 30 September 2019

Accepted: 11 February 2020

Published: 13 March 2020

Citation:

Kozuleva MA, Ivanov BN

Vetoshkina $D V$ and

Borisova-Mubarakshina MM (2020)

Minimizing an Electron Flow to

Molecular Oxygen in Photosynthetic

Electron Transfer Chain: An

Evolutionary View.

Front. Plant Sci. 11:211.

doi: 10.3389/fp/s.2020.00211
Recruitment of $\mathrm{H}_{2} \mathrm{O}$ as the final donor of electrons for light-governed reactions in photosynthesis has been an utmost breakthrough, bursting the evolution of life and leading to the accumulation of $\mathrm{O}_{2}$ molecules in the atmosphere. $\mathrm{O}_{2}$ molecule has a great potential to accept electrons from the components of the photosynthetic electron transfer chain (PETC) (so-called the Mehler reaction). Here we overview the Mehler reaction mechanisms, specifying the changes in the structure of the PETC of oxygenic phototrophs that probably had occurred as the result of evolutionary pressure to minimize the electron flow to $\mathrm{O}_{2}$. These changes are warranted by the fact that the efficient electron flow to $\mathrm{O}_{2}$ would decrease the quantum yield of photosynthesis. Moreover, the reduction of $\mathrm{O}_{2}$ leads to the formation of reactive oxygen species (ROS), namely, the superoxide anion radical and hydrogen peroxide, which cause oxidative stress to plant cells if they are accumulated at a significant amount. From another side, hydrogen peroxide acts as a signaling molecule. We particularly zoom in into the role of photosystem I (PSI) and the plastoquinone (PQ) pool in the Mehler reaction.

Keywords: photosystems, evolution, plastoquinone, phylloquinone, oxygen, reactive oxygen species

\section{INTRODUCTION}

Mehler reaction is the major source of reactive oxygen species (ROS), such as $\mathrm{O}_{2}{ }^{\bullet-}$ and $\mathrm{H}_{2} \mathrm{O}_{2}$, in chloroplasts. During the Mehler reaction, $\mathrm{O}_{2}$ molecules serve as an alternative electron acceptor from the photosynthetic electron transfer chain (PETC), being a safety valve to release surplus electrons and thus alleviating the PETC over-reduction. This reaction also contributes to building up of $\Delta \mathrm{pH}$ across the thylakoid membrane and produces a signaling messenger, $\mathrm{H}_{2} \mathrm{O}_{2}$, which is capable of initiating various signaling pathways (Ivanov et al., 2012). However, an efficient electron flow to $\mathrm{O}_{2}$ would decrease the photosynthetic quantum yield. Moreover, ROS, if not neutralized efficiently, lead to oxidative damage. Thus, the PETC evolution could have been guided toward minimizing and/or taking strong control over the Mehler reaction. 
Most of the PETC components were proposed as sites of $\mathrm{O}_{2}{ }^{\bullet-}$ photoproduction, the first step of the Mehler reaction. Among them, there are water-soluble and water-exposed components (Figure 1, open circles) and the components situated in hydrophobic zones (Figure 1, closed circles). The former produce $\mathrm{O}_{2} \bullet-$ in water bulk phases, e.g., stroma, while the latter produce $\mathrm{O}_{2}{ }^{\bullet-}$, which can be detected outside the membrane when diffused there or can be detected within the thylakoid membranes (Kozuleva et al., 2011). The value of $E_{m}$ $\left(\mathrm{O}_{2} / \mathrm{O}_{2}{ }^{\bullet-}\right)$ in water is $-160 \mathrm{mV}$, while in hydrophobic zones of proteins and membranes it is more negative, approximately $-550 \mathrm{mV}$ (Wardman, 1990). Only few components in PETC possess enough negative $E_{m}$ for $\mathrm{O}_{2}$ reduction within a thylakoid membrane. Numerous experiments unambiguously demonstrated that photosystem I (PSI) is the major site of $\mathrm{O}_{2}{ }^{\bullet-}$ photoproduction (Kozuleva and Ivanov, 2016). $\mathrm{O}_{2}^{\bullet-}$ generation by other components was shown under the disturbed PETC function. The second step of the Mehler reaction is $\mathrm{H}_{2} \mathrm{O}_{2}$ production via $\mathrm{O}_{2}{ }^{\bullet-}$ dismutation in stroma as catalyzed by superoxide dismutase. Apart from $\mathrm{O}_{2}{ }^{\bullet-}$ dismutation, another mechanism was shown to operate in the thylakoid membranes (Mubarakshina et al., 2006). It involves $\mathrm{O}_{2}{ }^{\bullet-}$ reduction by the plastoquinone (PQ) pool, namely, by plastoquinol $\left(\mathrm{PQH}_{2}\right)$ (Borisova-Mubarakshina et al., 2019). Thus, the Mehler reaction proceeds at a variety of sites, still leading to $\mathrm{O}_{2}{ }^{\bullet-}$ and subsequent $\mathrm{H}_{2} \mathrm{O}_{2}$ production.

The evolution of various photosynthetic complexes has been a subject of several recent reviews (Jagannathan et al., 2012; Rutherford et al., 2012; Pierella Karlusich and Carrillo, 2017; Orf et al., 2018). Here, we briefly summarize the structural changes which could have happened in PETC to control and minimize an electron flow to $\mathrm{O}_{2}$. The general evolutionary trends could include: (i) kinetic control, making the forward reactions faster than the competing electron flow to $\mathrm{O}_{2}$, (ii) redox tuning of cofactors, disabling spontaneous exergonic reactions with $\mathrm{O}_{2}$, and (iii) shielding of cofactors with protein environment, restricting $\mathrm{O}_{2}$ accessibility (Rutherford et al., 2012).

\section{PHOTOSYSTEM I}

All secondary electron acceptor cofactors of PSI were proposed as the sites of $\mathrm{O}_{2}$ photoreduction. The terminal FeS clusters $\mathrm{F}_{A} / \mathrm{F}_{B}$ are inevitably oxidized by $\mathrm{O}_{2}$ in the absence of ferredoxin (Fd). The role of intermediate cofactor $\mathrm{FeS}$ cluster $\mathrm{F}_{X}$ was claimed in Takahashi and Asada (1988) based on experiments showing that the primary $\mathrm{H}_{2} \mathrm{O}_{2}$ photoproduction site was a PsaA/PsaB heterodimer, which harbors $\mathrm{F}_{X}$. However, the PsaA/PsaB heterodimer also binds two phylloquinone (PhQ) molecules at the $A_{1}$ sites and they could also contribute to $\mathrm{H}_{2} \mathrm{O}_{2}$ photoproduction. For the first time, the role of PhQs was proposed by Kruk with coauthors (Kruk et al., 2003) since adding PhQ to quinone-depleted thylakoid membranes re-established the $\mathrm{O}_{2}$ uptake at a single light flash. This result still does not rule out that FeS clusters reduce $\mathrm{O}_{2}$ by electrons from $\mathrm{P}_{700}$ via $\mathrm{PhQ}$ re-incorporated to the $\mathrm{A}_{1}$ sites. The PhQ involvement in $\mathrm{O}_{2}$ photoreduction in intact PSI under steady-state illumination was
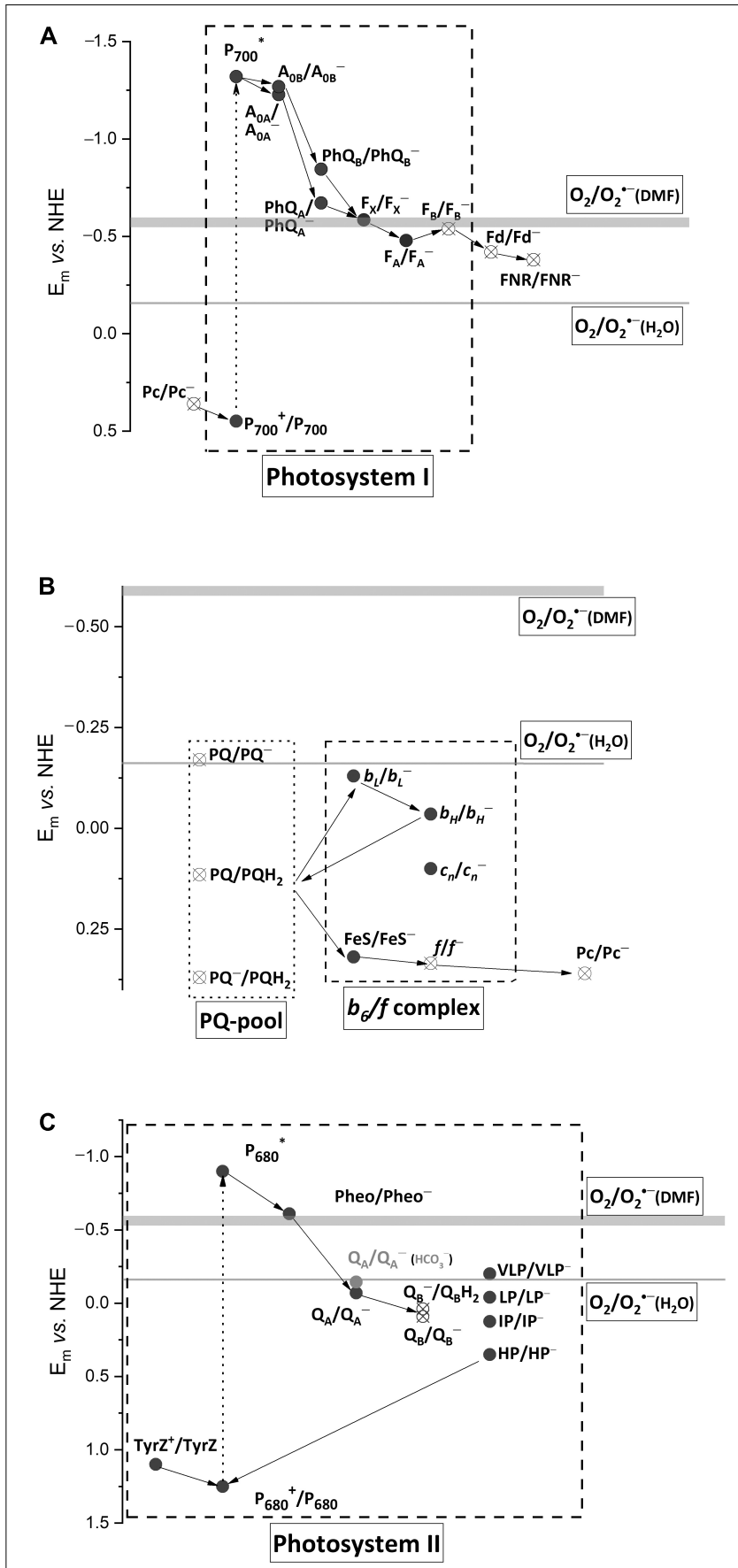

FIGURE 1 | Schematic diagram of forward electron transfer in PSI (A), PQ-pool and cytochrome $b_{6} / f$ complex (B), and PSII (C), with $E_{m}$ values of the cofactors. The $E_{\mathrm{m}}$ values of $\left(\mathrm{O}_{2} / \mathrm{O}_{2}{ }^{--}\right)$in water, $-160 \mathrm{mV}$, and dimethylformamide (DMF), from -550 to $-600 \mathrm{mV}$, are shown by thin and thick horizontal lines, respectively. Pc, plastocyanin; $\mathrm{P}_{700}$, the dimer of $\mathrm{Chl} \mathrm{a}$ molecules in PSI; $\mathrm{A}_{0}$, the primary electron acceptor in PSI; $\mathrm{PhQ}$,

phylloquinone, a secondary electron acceptor in PSI; $\mathrm{F}_{X}$, a 4Fe-4S cluster, a secondary electron acceptor in $\mathrm{PSI} ; \mathrm{F}_{\mathrm{A}}$ and $\mathrm{F}_{\mathrm{B}}$, 4Fe-4S clusters, the terminal electron acceptors in PSI; Fd, ferredoxin, the mobile electron acceptor; FNR, ferredoxin/ $\mathrm{NADP}^{+}$reductase; $\mathrm{PQ}, \mathrm{PQ}^{\bullet-}$, and $\mathrm{PQH}_{2}$, plastoquinone, plastosemiquinone, and plastoquinol; FeS, a $2 \mathrm{Fe}-2 \mathrm{~S}$ cluster of Rieske protein; $f$, cytochrome $\mathrm{f} ; b_{L}$ and $b_{H}$, the low- and high-potential forms of cytochrome (Continued) 


\section{FIGURE 1 | Continued}

$b_{6} ; c_{n}$, the heme covalently bound to cytochrome $b_{6}$; Tyr $Z$, the redox active tyrosine residue; $\mathrm{P}_{680}$, the dimer of $\mathrm{Chl}$ a molecules in PSIl; Pheo, pheophytin, a primary electron acceptor in PSII; $Q_{A}$, the tightly bound plastoquinone in PSII, the secondary electron acceptor in PSII; $Q_{B}$, the loosely bound plastoquinone in PSII, the terminal electron acceptor in PSII; VLP, LP, IP, and $\mathrm{HP}$, the very low-, low-, intermediate-, and high-potential forms of cytochrome $b_{559}$ in PSII. The $E_{m}$-values for PSI cofactors are according to Ptushenko et al. (2008), those for $b_{6} / f$ complex are according to Alric et al. (2005), those for PSIl are according to Brinkert et al. (2016) and Causmaecker et al. (2019), except cytochrome $b_{559}$ (Khorobrykh, 2019). Closed circles, components situated in hydrophobic zones; open circles, water bulk phases exposed components.

proposed based on comparing $\mathrm{O}_{2}$ photoreduction as a function of irradiance in the wild-type PSI with that in the mutant PQcontaining PSI (Kozuleva et al., 2014). The authors concluded that the PhQs at the $A_{1}$ sites are the major contributor to $\mathrm{O}_{2}{ }^{\bullet-}$ generation.

From an evolutionary point of view, the terminal cofactor $\mathrm{F}_{B}$ can be one of the sites where the Mehler reaction should have been taken under control. This cofactor possesses negative $E_{m}$, allowing for the efficient reduction of both $\mathrm{Fd}$ and $\mathrm{O}_{2}$. However, $\mathrm{Fd}$ is a mobile protein, diffusing to and out of PSI and leaving $\mathrm{F}_{B}{ }^{-}$transiently open to $\mathrm{O}_{2}$. If $\mathrm{F}_{B}{ }^{-}$is oxidized by $\mathrm{O}_{2}$ efficiently, it would be insufficient in a steady-state reduction of Fd. However, the electron lives mostly on $\mathrm{F}_{A}$, not $\mathrm{F}_{B}$, because of a positive shift of $E_{m}\left(\mathrm{~F}_{A} / \mathrm{F}_{A}{ }^{-}\right)$relative to $\left(\mathrm{F}_{B} / \mathrm{F}_{B}{ }^{-}\right)$(Figure 1A; Fischer et al., 1997; Shinkarev et al., 2000). $\mathrm{F}_{A}$ is embedded deeper in the protein, which shields it from $\mathrm{O}_{2}$. This feature allows keeping of electrons for $\mathrm{Fd}$ and avoiding any wasteful electron leakage to $\mathrm{O}_{2}$.

The PsaC protein carrying $\mathrm{F}_{A}$ and $\mathrm{F}_{B}$ is homologous to mobile ferredoxins in anoxigenic phototrophs (Jagannathan and Golbeck, 2009). It is widely accepted that, during evolution, the ancestral mobile Fd was tightly bound to the ancestral homodimeric reaction center (RC). This binding resulted in an elongation of the ET chain in the RC that could have aimed at stabilizing the charge separation state and minimizing the charge recombination, which could lead to ${ }^{3} \mathrm{P}_{700}$ and, hence, ${ }^{1} \mathrm{O}_{2}$ formation (Orf et al., 2018). However, that binding probably provided an additional protein shielding for $\mathrm{F}_{X}$, which was the terminal cofactor in the ancestral $\mathrm{RC}$, and for $\mathrm{F}_{A}$, limiting $\mathrm{O}_{2}$ diffusion and preventing unproductive electron leakage (Jagannathan et al., 2012). The protein shielding of these FeS clusters, being potentially capable of catalyzing $\mathrm{H}_{2} \mathrm{O}_{2}$ decomposition into a highly reactive $\mathrm{HO}^{\bullet}$ (Šnyrychová et al., 2006), could have additionally protected the PSI acceptor side from $\mathrm{HO}^{\bullet}$ formation.

Binding of the ancestral $\mathrm{Fd}$ to the ancestral homodimeric $\mathrm{RC}$ resulted in $\mathrm{RC}$ asymmetry through locating the $\mathrm{F}_{A}$ cluster closer to one of the quinones $\left(\mathrm{PhQ}_{B}\right)$, bringing about a negative shift in $E_{m}\left(\mathrm{PhQ}_{B} / \mathrm{PhQ}_{B}{ }^{\bullet-}\right)$ (Rutherford et al., 2012). The difference in $E_{m}$ between $\mathrm{PhQ}_{A}$ and $\mathrm{PhQ}_{B}$ is up to $170 \mathrm{mV}$ (Ptushenko et al., 2008). Rutherford with coauthors presented an elegant hypothesis explaining the benefit of this asymmetry as it eliminates ${ }^{3} \mathrm{P}_{700}$ (and hence ${ }^{1} \mathrm{O}_{2}$ ) formation under the conditions of the Fd pool over-reduction (Rutherford et al., 2012). In line with this hypothesis, $\mathrm{PhQ}^{\bullet-}$ oxidation by $\mathrm{O}_{2}$ sustains a forward
ET and contributes to both alleviating PETC over-reduction and preventing charge recombination (Kozuleva and Ivanov, 2016). Both PhQs in PSI have one of the most negative $E_{m}$ in the PETC ( -671 and $-840 \mathrm{mV}$ for $\mathrm{PhQ}_{A}$ and $\mathrm{PhQ}_{B}$, respectively; Figure 1A), which allows phyllosemiquinones to reduce $\mathrm{O}_{2}$ even in the hydrophobic zones of the thylakoid membranes, where $E_{m}\left(\mathrm{O}_{2} / \mathrm{O}_{2}{ }^{\bullet-}\right)$ is close to $-550 \mathrm{mV}$ (see above). Due to a longer lifetime, $\mathrm{PhQ}_{A}{ }^{\bullet-}$ gets higher chances to react with $\mathrm{O}_{2}$, although the more negative $E_{m}$ of $\mathrm{PhQ}_{B} / \mathrm{PhQ}_{B}{ }^{\bullet-}$ provides a larger $-\Delta G$ in reaction with $\mathrm{O}_{2}$. However, the particular impact of each $\mathrm{PhQ}$ as well as clarifying the $\mathrm{F}_{X}$ role is still open questions.

\section{FERREDOXIN AND FNR}

In bacterial type $\mathrm{Fd}$, two $4 \mathrm{Fe}-4 \mathrm{~S}$ clusters are partially exposed to solvent and accessible for $\mathrm{O}_{2}$ attacks (Jagannathan et al., 2012). After binding the ancestral $\mathrm{Fd}$ to $\mathrm{RC}$, the organisms recruited another $\mathrm{Fd}$, where a single $2 \mathrm{Fe}-2 \mathrm{~S}$ cluster is shielded by a protein.

A long-lasting controversy on the role of Fd in the Mehler reaction was solved nearly a decade ago. In the absence of $\mathrm{NADP}^{+}$, which is the major electron sink for $\mathrm{Fd}, \mathrm{O}_{2}$ inevitably oxidizes the reduced $\mathrm{Fd}\left(\mathrm{Fd}^{-}\right)$. In the presence of $\mathrm{NADP}^{+}$, simultaneously with its photoreduction, the electron flow to $\mathrm{O}_{2}$ was shown to be significant in high light; however, the contribution of $\mathrm{Fd}$ was almost negligible relative to that of the membrane-bound PETC components (Kozuleva and Ivanov, 2010). These results reveal a low reactivity of $\mathrm{Fd}^{-}$toward $\mathrm{O}_{2}$, which enables Fd to fulfill the function of stromal hub-donating electrons to multiple enzymes and proteins, including ferredoxin$\mathrm{NADP}^{+}$reductase (FNR) (Hanke and Mulo, 2013).

The Fd affinity to its redox partners, i.e., PSI acceptor side, was also raised to ensure the competition with $\mathrm{O}_{2}$ for electrons. However, this is not entirely the case of FNR. Although a semiquinone form of FAD prosthetic group in FNR can react with $\mathrm{O}_{2}$ (Massey, 1994), so far there are no reliable experimental data demonstrating that $\mathrm{FNR}$ is involved in $\mathrm{O}_{2}$ photoreduction in the thylakoid membranes (Kozuleva and Ivanov, 2016). The FNR of oxygenic phototrophs possesses $\sim 10$ times higher catalytic activity than the bacterial FNR (Pierella Karlusich and Carrillo, 2017), with affinity remaining roughly the same. The high catalytic activity is likely achieved through conformational changes caused by $\mathrm{NADP}^{+}$binding to $\mathrm{FNR}$, which greatly facilitate both the $\mathrm{Fd}^{-}$oxidation (Batie and Kamin, 1984) and the liberation of the oxidized Fd from the complex (Mulo and Medina, 2017). This enhancement in the FNR catalytic activity most possibly decreased the chances for both the FAD semiquinone $\left(\mathrm{Q}^{\bullet-}\right)$ oxidation by $\mathrm{O}_{2}$ and the formation of $\mathrm{Fd}: \mathrm{FNR}^{\bullet-}$ complex in the absence of $\mathrm{NADP}^{+}$.

\section{PLASTOQUINONE POOL}

$\mathrm{O}_{2}{ }^{\bullet-}$ photoproduction by $\mathrm{PQ}^{\bullet-}$ in the $\mathrm{PQ}$ pool was demonstrated (Khorobrykh and Ivanov, 2002; Vetoshkina et al., 2017). However, the maximal $\mathrm{O}_{2}{ }^{\bullet-}$ production rates observed in the pool were 10 times lower than in the PSI. 
While anoxygenic phototrophs use menaquinone (MQ) and ubiquinone (UQ), the oxygenic ones recruited $P Q$, a representative of a "more recent" group of quinones (SchoeppCothenet et al., 2009). MQ was probably the first quinone in ancient photosynthetic membranes. The rationale for replacing MQ with PQ is clear: the $E_{m}$ values of $\left(\mathrm{Q} / \mathrm{Q}^{\bullet-}\right)$ and $\left(\mathrm{Q} / \mathrm{QH} \mathrm{H}_{2}\right)$ are $\sim 100 \mathrm{mV}$ (Kishi et al., 2017) and $\sim 180 \mathrm{mV}$ (Bergdoll et al., 2016), more negative for MQ than for PQ (Figure 1B). This means that $\mathrm{PQ}^{\bullet-}$ and the PQ pool itself in the reduced state are more stable in the presence of $\mathrm{O}_{2}$. Furthermore, $\mathrm{pK}_{a}\left(\mathrm{Q}^{\bullet-} / \mathrm{QH}\right)$ for $\mathrm{PQ}$ is higher than for $M Q$, providing an easier protonation and, hence, a higher stability of plastosemiquinone (Hasegawa et al., 2017).

A possible rationale for choosing PQ instead of UQ in the PETC of oxygenic phototrophs is still vague. Firstly, the $\mathrm{O}_{2}{ }^{\bullet-}$ generation by free $\mathrm{UQ}^{\bullet-}$ in the mitochondria was discovered as early as in 80-s (Turrens et al., 1985). This reaction has long been considered as an important source of $\mathrm{O}_{2}{ }^{\bullet-}$ in animal cells. On the contrary, $\mathrm{PQ}^{\bullet-}$ in photosynthetic cells has little impact on $\mathrm{O}_{2}{ }^{\bullet-}$ production, as stated above. Secondly, $\mathrm{PQH}_{2}$ is more efficient as an antioxidant than $\mathrm{UQH}_{2}$ (Borisova-Mubarakshina et al., 2019), e.g., in lipid peroxidation prevention (Kruk et al., 1997). A consequence of higher antioxidant activity of $\mathrm{PQH}_{2}$ is its higher ability to reduce $\mathrm{O}_{2}^{\bullet-}$ to $\mathrm{H}_{2} \mathrm{O}_{2}$. It was shown that the PQ pool in the thylakoid membranes (presumably $\mathrm{PQH}_{2}$ ) is indeed oxidized by $\mathrm{O}_{2}^{\bullet-}$ (Borisova-Mubarakshina et al., 2018). Therefore, despite the low $\mathrm{O}_{2}{ }^{\bullet-}$-generating activity, the contribution of the PQ pool to the Mehler reaction can be essential due to the production of $\mathrm{H}_{2} \mathrm{O}_{2}$ from $\mathrm{O}_{2}{ }^{\bullet-}$. We hypothesize that ensuring the efficient transformation of $\mathrm{O}_{2}{ }^{\bullet-}$, which is generated by PSI, to $\mathrm{H}_{2} \mathrm{O}_{2}$ could be one of the evolutionary driving forces for the choice of PQ.

Replacing MQ with PQ as a mobile pool in the thylakoid membrane inevitably affected all of the complexes interacting with quinone. All cofactors in photosystem II (PSII) and cytochrome $b_{6} / f$ complexes have $110-150 \mathrm{mV}$ more positive $E_{m}$ values than in their MQ-based analogs (Schoepp-Cothenet et al., 2009; Bergdoll et al., 2016).

\section{CYTOCHROME $b_{6} / f$ COMPLEX}

The cytochrome $b_{6} / f$ complex is also considered to be an $\mathrm{O}_{2}$ photoreduction site (Taylor et al., 2018). The high $E_{m}$ values of the $b_{6} f$ complex cofactors are a consequence of MQ replacement with PQ (Bergdoll et al., 2016). Among its ET cofactors, the $b_{L}$ heme possesses one of the lowest $E_{m},-130 \mathrm{mV}$ (Alric et al., 2005). Thermodynamically, this heme can hardly reduce $\mathrm{O}_{2}$ since $E_{m}\left(\mathrm{O}_{2} / \mathrm{O}_{2}{ }^{\bullet-}\right)$ in the membrane is close to $-550 \mathrm{mV}$ (Figure 1B, see above). The fast ET from $b_{L}$ to $b_{H}$ decreases the possibility of a $b_{L}$ reaction with $\mathrm{O}_{2}$.

In several studies, $\mathrm{PQ}^{\bullet-}$ at the quinol-oxidizing $\left(\mathrm{Q}_{0}\right)$ site of the complex is considered as the electron donor to $\mathrm{O}_{2}$. However, the concerted oxidation of $\mathrm{PQH}_{2}$ diminishes the $\mathrm{PQ}^{\bullet-}$ lifetime. If semiquinone is produced, it is either quickly oxidized by $b_{L}$ heme or reduced by it, if the heme is pre-reduced. The dimer organization of the $b_{6} / f$ complex was proposed to lower the chances of $\mathrm{O}_{2}{ }^{\bullet-}$ generation at the $\mathrm{Q}_{o}$ site (Rutherford et al., 2012). In the $b c_{1}$ complex, a spin-spin complex state between the semiquinone and the Rieske cluster was shown to suppress $\mathrm{O}_{2}{ }^{\bullet-}$ generation (Bujnowicz et al., 2019). This keeps up well with the experimental observations that $\mathrm{PQ}^{\bullet-}$ can reduce $\mathrm{O}_{2}$ once it leaves the $\mathrm{Q}_{o}$ pocket (Forquer et al., 2006), becoming a part of the pool (see above). It was demonstrated that $\mathrm{O}_{2}{ }^{\bullet-}$ production by the isolated $b_{6} / f$ complexes was 10 times higher than the one by the isolated $b c_{1}$ complexes (Baniulis et al., 2013). This can be explained by an easier liberation of semiquinone from the $\mathrm{Q}_{0}$ site in the former case. It is important that, in vivo, such $\mathrm{PQ}^{\bullet-}$ would appear at the luminal side of the thylakoid membrane. The lumen $\mathrm{pH}$ determines the protonation of $\mathrm{PQ}^{\bullet-}$. Since $\mathrm{PQH}^{\bullet}$ has a lower chance to reduce $\mathrm{O}_{2}$, the lumen $\mathrm{pH}$ can regulate the $\mathrm{O}_{2}{ }^{\bullet-}$ production there.

The appearance of semiquinone at the quinone-reducing site $\left(\mathrm{Q}_{r}\right)$ of the $b c_{1}$ complex from purple bacteria was shown (Drachev et al., 1989). There are still no reliable data on semiquinone formation at the $\mathrm{Q}_{r}$ site of the $b_{6} / f$ complex. The double reduction of $\mathrm{PQ}$ occurs there when the second electron is transferred to the $b_{H}$ heme (Ivanov, 1993). The residence of the first electron at the $b_{H}$ heme can be a result of the $c_{n}$ heme situated in close vicinity to the $b_{H}$ in the $b_{6} / f$ complex.

\section{PHOTOSYSTEM II}

Three major tasks could have been solved during the evolution of PSII: (i) the existence of highly oxidizing $\mathrm{P} 680^{+}$, (ii) dealing with charge recombination leading to ${ }^{1} \mathrm{O}_{2}$ production, and (iii) stabilization of $\mathrm{Q}_{B}{ }^{-}$waiting for the second electron (Rutherford et al., 2012). $\mathrm{O}_{2}{ }^{\bullet-}$ production in PSII was shown many times (Pospíšil, 2012). Pheophytin (Pheo), $\mathrm{Q}_{A}, \mathrm{Q}_{B}$, and cytochrome $b_{559}$ were suggested as the sites of $\mathrm{O}_{2}$ reduction to $\mathrm{O}_{2}{ }^{\bullet-}$, based presumably on the experiments with PSII complexes with disrupted function, e.g., after modifications of the wateroxidizing complex.

Although Pheo- ${ }^{-}$possesses $E_{m},-610 \mathrm{mV}$ (Rappaport et al., 2002), negative enough to reduce $\mathrm{O}_{2}$ even in hydrophobic media (Figure 1C), its lifetime is rather short (300 ps) such that it prevents the electron leakage to $\mathrm{O}_{2}$. This reaction with $\mathrm{Q}_{A}^{-}$(Ivanov and Khorobrykh, 2003; Pospísil, 2012) is thermodynamically unfavorable due to a more positive $E_{m}\left(\mathrm{Q}_{A} / \mathrm{Q}_{A}^{-}\right),-70 \mathrm{mV}$ (Brinkert et al., 2016), than $E_{m}$ $\left(\mathrm{O}_{2} / \mathrm{O}_{2}{ }^{\bullet-}\right)$. However, the binding of $\mathrm{HCO}_{3}{ }^{-}$to non-heme $\mathrm{Fe}$ situated between the $\mathrm{Q}_{A}$ and the $\mathrm{Q}_{B}$ shifts $E_{m}\left(\mathrm{Q}_{A} / \mathrm{Q}_{A}{ }^{-}\right)$ to $-145 \mathrm{mV}$, making the electron leakage from $\mathrm{Q}_{A}^{-}$to $\mathrm{O}_{2}$ more probable. In contrast to $\mathrm{Q}_{A}, \mathrm{Q}_{B}$ undergoes two sequential reduction steps, meaning that $\mathrm{Q}_{B}{ }^{-}$lives for a longer time waiting for the second electron. However, $\mathrm{Q}_{B}{ }^{-}$ is thermodynamically stable due to the positive $E_{m}$ potentials (Causmaecker et al., 2019).

The role of a very low potential form of cytochrome $b_{559}\left(E_{m}\right.$ is -150 to $\left.-200 \mathrm{mV}\right)$ in $\mathrm{O}_{2}$ reduction was also proposed (Khorobrykh, 2019). However, the fraction of this form is extremely small under normal conditions and increases only when the PSII functioning is severely perturbed. The $b_{559}$ heme is embedded in the hydrophobic zone of the membrane; therefore, $\mathrm{O}_{2}$ photoreduction by $b_{559}$ heme is thermodynamically unfavorable. 


\section{DISCUSSION}

In this review, we briefly summarize some features of the modern PETC, which have evolved at the background of the Mehler reaction. The main site of $\mathrm{O}_{2}{ }^{\bullet-}$ generation is PSI. Several experiments revealed that PhQ could be the major contributor to this process (Kruk et al., 2003; Kozuleva et al., 2011, 2014). The reactivity of the $\mathrm{FeS}$ components with $\mathrm{O}_{2}$, especially $\mathrm{F}_{B}$ and $\mathrm{Fd}$, was diminished by redox tuning and protein shielding. The recruitment of a high-potential $\mathrm{PQ}$ to the membrane quinone pool instead of a low-potential MQ was driven by the necessity to keep the pool in the reduced state under illumination in the presence of $\mathrm{O}_{2}$. Replacing MQ with PQ triggered a redox tuning of PSII and cytochrome $b_{6} / f$ complex cofactors, disabling, among other things, efficient $\mathrm{O}_{2}{ }^{\bullet-}$ generation in these complexes. The only MQ-based cofactor preserved in the modern PETC is PhQ, which is likely to be the main site of $\mathrm{O}_{2}{ }^{\bullet-}$ generation.

The stromal production of $\mathrm{O}_{2}{ }^{\bullet-}$ via $\mathrm{Fd}$ greatly increases if the $\mathrm{NADP}^{+}$recovering in the Calvin-Benson-Bassham cycle is retarded, e.g., due to closed stomata. In the stroma, $\mathrm{H}_{2} \mathrm{O}_{2}$ is produced from $\mathrm{O}_{2}{ }^{\bullet-}$ under catalysis by superoxide dismutase. $\mathrm{O}_{2}$ reduction by $\mathrm{PhQ}^{\bullet-}$ can account for $\mathrm{O}_{2}{ }^{\bullet-}$ appearance within the thylakoid membrane (Kozuleva et al., 2011); however, a significant part of $\mathrm{O}_{2}{ }^{\bullet-}$ formed by $\mathrm{PhQ}^{\bullet-}$ still likely diffuses outside the membrane. Nevertheless, the increasing irradiance resulted in both a larger $\mathrm{O}_{2}{ }^{\bullet-}$ production just within the thylakoid membrane and a larger $\mathrm{H}_{2} \mathrm{O}_{2}$ production via $\mathrm{O}_{2}{ }^{\bullet-}$ reduction by $\mathrm{PQH}_{2}$, i.e., by the mechanism different from dismutation (Borisova-Mubarakshina et al., 2012).

Thus, in chloroplasts, $\mathrm{H}_{2} \mathrm{O}_{2}$ is produced via two distinct reactions in two distinct chloroplast compartments. We believe that this observation may be important for the understanding of $\mathrm{H}_{2} \mathrm{O}_{2}$-mediated signal transduction. The stromal $\mathrm{H}_{2} \mathrm{O}_{2}$, which might be considered as a messenger of $\mathrm{NADP}^{+} / \mathrm{NADPH}$ status, can oxidize thioredoxins (Hofmann, 2010; Netto and

\section{REFERENCES}

Alric, J., Pierre, Y., Picot, D., Lavergne, J., and Rappaport, F. (2005). Spectral and redox characterization of the heme ci of the cytochrome b6f complex. Proc. Natl. Acad. Sci. U.S.A. 102, 15860-15865. doi: 10.1073/pnas.050810 2102

Baniulis, D., Hasan, S. S., Stofleth, J. T., and Cramer, W. A. (2013). Mechanism of enhanced superoxide production in the cytochrome b6f complex of oxygenic photosynthesis. Biochemistry 52, 8975-8983. doi: 10.1021/bi4013534

Batie, C. J., and Kamin, H. (1984). Ferredoxin:NADP (oxidoreductase. Equilibria in binary and ternary complexes with NADP (and ferredoxin. J. Biol. Chem. 259, 8832-8839.

Bergdoll, L., ten Brink, F., Nitschke, W., Picot, D., and Baymann, F. (2016). From low- to high-potential bioenergetic chains: Thermodynamic constraints of Q-cycle function. Biochim. Biophys. Acta 1857, 1569-1579. doi: 10.1016/j. bbabio.2016.06.006

Borisova-Mubarakshina, M. M., Ivanov, B. N., Vetoshkina, D. V., Lubimov, V. Y., Fedorchuk, T. P., Naydov, I. A., et al. (2015). Long-term acclimatory response to excess excitation energy: evidence for a role of hydrogen peroxide in the regulation of photosystem II antenna size. J. Exp. Bot. 66, 7151-7164. doi: 10.1093/jxb/erv410

Borisova-Mubarakshina, M. M., Kozuleva, M. A., Rudenko, N. N., Naydov, I. A., Klenina, I. B., and Ivanov, B. N. (2012). Photosynthetic electron flow to oxygen and diffusion of hydrogen peroxide through the chloroplast envelope
Antunes, 2016). Therefore, a temporary $\mathrm{H}_{2} \mathrm{O}_{2}$ accumulation in the stroma can affect the expression of chloroplast genes and/or the translation aimed at the fast adaptation of photosynthetic apparatus. $\mathrm{H}_{2} \mathrm{O}_{2}$ formed by the membrane $\mathrm{PQ}$ pool might be considered as a messenger of PETC status. It is important in terms of the PQ pool function as a central hub, of which the redox state represents a signal for both the chloroplast gene expression (Pfannschmidt et al., 2009) and the retrograde signaling pathways from the chloroplast to the nucleus (Pfannschmidt et al., 2003). For example, the PQ pool redox state initiates the changes in the PSII light-harvesting antenna size as a long-term acclimation to light conditions (Escoubas et al., 1995; Frigerio et al., 2007). We demonstrated that it is $\mathrm{H}_{2} \mathrm{O}_{2}$ rather than the PQ pool reduction state itself that is responsible for the antenna size reduction in high light (Borisova-Mubarakshina et al., 2015, 2019). Therefore, we suppose that a high potential of the PQ pool to form $\mathrm{H}_{2} \mathrm{O}_{2}$ in high light and under stress conditions could serve as evolutionarily set to signal about the PETC redox state to adjust to the ever-changing environmental conditions.

\section{AUTHOR CONTRIBUTIONS}

MK and MB-M designed the concept of the article. All authors contributed to the writing of the first draft and manuscript revision, and approved the submitted version. MK incorporated all inputs from the coauthors, reviewers, and editor.

\section{FUNDING}

This work was funded by the Russian Science Foundation, project 17-14-01371 and by The Ministry of Science and Higher Education of the Russian Federation, State Scientific Program, theme no. AAAA-A17-117030110135-1.

via aquaporins. Biochim. Biophys. Acta 1817, 1314-1321. doi: 10.1016/j.bbabio. 2012.02.036

Borisova-Mubarakshina, M. M., Naydov, I. A., and Ivanov, B. N. (2018). Oxidation of the plastoquinone pool in chloroplast thylakoid membranes by superoxide anion radicals. FEBS Lett. 592, 3221-3228. doi: 10.1002/1873-3468.13237

Borisova-Mubarakshina, M. M., Vetoshkina, D. V., and Ivanov, B. N. (2019). Antioxidant and signaling functions of the plastoquinone pool in higher plants. Physiol. Plant. 166, 181-198. doi: 10.1111/ppl.12936

Brinkert, K., Causmaecker, S. D., Krieger-Liszkay, A., Fantuzzi, A., and Rutherford, A. W. (2016). Bicarbonate-induced redox tuning in Photosystem II for regulation and protection. PNAS 113, 12144-12149. doi: 10.1073/pnas. 1608862113

Bujnowicz, Ł, Borek, A., Kuleta, P., Sarewicz, M., and Osyczka, A. (2019). Suppression of superoxide production by a spin-spin coupling between semiquinone and the Rieske cluster in cytochrome bc1. FEBS Lett. 593, 3-12. doi: 10.1002/1873-3468.13296

Causmaecker, S. D., Douglass, J. S., Fantuzzi, A., Nitschke, W., and Rutherford, A. W. (2019). Energetics of the exchangeable quinone, QB, in Photosystem II. PNAS 116, 19458-19463. doi: 10.1073/pnas.1910675116

Drachev, L. A., Kaurov, B. S., Mamedov, M. D., Mulkidjanian, A. Y., Semenov, A. Yu, Shinkarev, V. P., et al. (1989). Flash-induced electrogenic events in the photosynthetic reaction center and bcl complexes of Rhodobacter sphaeroides chromatophores. Biochim. Biophys. Acta 973, 189-197. doi: 10.1016/S00052728(89)80421-9 
Escoubas, J. M., Lomas, M., LaRoche, J., and Falkowski, P. G. (1995). Light intensity regulation of $\mathrm{cab}$ gene transcription is signaled by the redox state of the plastoquinone pool. PNAS 92, 10237-10241. doi: 10.1073/pnas.92.22. 10237

Fischer, N., Sétif, P., and Rochaix, J.-D. (1997). Targeted mutations in the psac gene of Chlamydomonas reinhardtii: preferential reduction of $\mathrm{FB}$ at low temperature is not accompanied by altered electron flow from Photosystem I to Ferredoxin. Biochemistry 36, 93-102. doi: 10.1021/bi962244v

Forquer, I., Covian, R., Bowman, M. K., Trumpower, B. L., and Kramer, D. M. (2006). Similar transition states mediate the Q-cycle and superoxide production by the cytochrome bc1 complex. J. Biol. Chem. 281, 38459-38465. doi: 10.1074/ jbc.M605119200

Frigerio, S., Campoli, C., Zorzan, S., Fantoni, L. I., Crosatti, C., Drepper, F., et al. (2007). Photosynthetic antenna size in higher plants is controlled by the plastoquinone redox state at the post-transcriptional rather than transcriptional level. J. Biol. Chem. 282, 29457-29469. doi: 10.1074/jbc.M70513 2200

Hanke, G., and Mulo, P. (2013). Plant type ferredoxins and ferredoxindependent metabolism. Plant Cell Environ. 36, 1071-1084. doi: 10.1111/pce. 12046

Hasegawa, R., Saito, K., Takaoka, T., and Ishikita, H. (2017). pKa of ubiquinone, menaquinone, phylloquinone, plastoquinone, and rhodoquinone in aqueous solution. Photosynth. Res. 133, 297-304. doi: 10.1007/s11120-017-0382-y

Hofmann, N. R. (2010). A new thioredoxin is involved in plastid gene expression. Plant Cell 22:1423. doi: 10.1105/tpc.110.220512

Ivanov, B., and Khorobrykh, S. (2003). Participation of photosynthetic electron transport in production and scavenging of reactive oxygen species. Antioxid. Redox Signal. 5, 43-53. doi: 10.1089/152308603321223531

Ivanov, B., Kozuleva, M., and Mubarakshina, M. (2012). "Oxygen metabolism in chloroplast," in Cell Metabolism-Cell Homeostasis and Stress Response, ed. P. Bubulya (London: InTech).

Ivanov, B. N. (1993). "Stoichiometry of proton uptake by thylakoids during electron transport in chloroplasts," in Photosynthesis: Photoreactions to Plant Productivity, eds Y. P. Abrol and P. Mohanty (Dordrecht: Springer Netherlands), 109-128. doi: 10.1007/978-94-011-2708-0_4

Jagannathan, B., and Golbeck, J. H. (2009). Understanding of the binding interface between PsaC and the PsaA/PsaB heterodimer in Photosystem I. Biochemistry 48, 5405-5416. doi: 10.1021/bi900243f

Jagannathan, B., Shen, G., and Golbeck, J. H. (2012). “The evolution of type i reaction centers: the response to oxygenic photosynthesis," in Functional Genomics and Evolution of Photosynthetic Systems Advances in Photosynthesis and Respiration, (Dordrecht: Springer), 285-316. doi: 10.1007/978-94-0071533-2 12

Khorobrykh, A. (2019). Hydrogen peroxide and superoxide anion radical photoproduction in PSII preparations at various modifications of the wateroxidizing complex. Plants 8:329. doi: 10.3390/plants8090329

Khorobrykh, S. A., and Ivanov, B. N. (2002). Oxygen reduction in a plastoquinone pool of isolated pea thylakoids. Photosynth. Res. 71, 209-219. doi: 10.1023/A: 1015583502345

Kishi, S., Saito, K., Kato, Y., and Ishikita, H. (2017). Redox potentials of ubiquinone, menaquinone, phylloquinone, and plastoquinone in aqueous solution. Photosynth. Res. 134, 193-200. doi: 10.1007/s11120-017-0433-4

Kozuleva, M., Klenina, I., Proskuryakov, I., Kirilyuk, I., and Ivanov, B. (2011). Production of superoxide in chloroplast thylakoid membranes: ESR study with cyclic hydroxylamines of different lipophilicity. FEBS Lett. 585, 1067-1071. doi: 10.1016/j.febslet.2011.03.004

Kozuleva, M. A., and Ivanov, B. N. (2010). Evaluation of the participation of ferredoxin in oxygen reduction in the photosynthetic electron transport chain of isolated pea thylakoids. Photosynth. Res. 105, 51-61. doi: 10.1007/s11120010-9565-5

Kozuleva, M. A., and Ivanov, B. N. (2016). The mechanisms of oxygen reduction in the terminal reducing segment of the chloroplast photosynthetic electron transport chain. Plant Cell Physiol. 57, 1397-1404. doi: 10.1093/pcp/pcw035

Kozuleva, M. A., Petrova, A. A., Mamedov, M. D., Semenov, A. Y., and Ivanov, B. N. (2014). O2 reduction by photosystem I involves phylloquinone under steadystate illumination. FEBS Lett. 588, 4364-4368. doi: 10.1016/j.febslet.2014. 10.003
Kruk, J., Jemioła-Rzemiłska, M., Burda, K., Schmid, G. H., and Strzałka, K. (2003). scavenging of superoxide generated in Photosystem I by Plastoquinol and other Prenyllipids in Thylakoid membranes. Biochemistry 42, 8501-8505. doi: 10.1021/bi034036q

Kruk, J., Jemioła-Rzemińska, M., and Strzałka, K. (1997). Plastoquinol and (tocopherol quinol are more active than ubiquinol and (-tocopherol in inhibition of lipid peroxidation. Chem. Phys. Lipids 87, 73-80. doi: 10.1016/S00093084(97)00027-3

Massey, V. (1994). Activation of molecular oxygen by flavins and flavoproteins. J. Biol. Chem. 269, 22459-22462.

Mubarakshina, M., Khorobrykh, S., and Ivanov, B. (2006). Oxygen reduction in chloroplast thylakoids results in production of hydrogen peroxide inside the membrane. Biochim. Biophys. Acta 1757, 1496-1503. doi: 10.1016/j.bbabio. 2006.09.004

Mulo, P., and Medina, M. (2017). Interaction and electron transfer between ferredoxin-NADP (oxidoreductase and its partners: structural, functional, and physiological implications. Photosynth. Res. 134, 265-280. doi: 10.1007/s11120017-0372-0

Netto, L. E. S., and Antunes, F. (2016). The roles of peroxiredoxin and thioredoxin in hydrogen peroxide sensing and in signal transduction. Mol. Cells 39, 65-71. doi: $10.14348 /$ molcells.2016.2349

Orf, G. S., Gisriel, C., and Redding, K. E. (2018). Evolution of photosynthetic reaction centers: insights from the structure of the heliobacterial reaction center. Photosynth. Res. 138, 11-37. doi: 10.1007/s11120-0180503-2

Pfannschmidt, T., Bräutigam, K., Wagner, R., Dietzel, L., Schröter, Y., Steiner, S., et al. (2009). Potential regulation of gene expression in photosynthetic cells by redox and energy state: approaches towards better understanding. Ann. Bot. 103, 599-607. doi: 10.1093/aob/mcn081

Pfannschmidt, T., Schütze, K., Fey, V., Sherameti, I., and Oelmüller, R. (2003). Chloroplast redox control of nuclear gene expression-a new class of plastid signals in interorganellar communication. Antioxid. Redox Signal. 5, 95-101. doi: $10.1089 / 152308603321223586$

Pierella Karlusich, J. J., and Carrillo, N. (2017). Evolution of the acceptor side of photosystem I: ferredoxin, flavodoxin, and ferredoxin-NADP (oxidoreductase. Photosyn. Res. 134, 235-250. doi: 10.1007/s11120-0170338-2

Pospíšil, P. (2012). Molecular mechanisms of production and scavenging of reactive oxygen species by photosystem II. Biochim. Biophys. Acta 1817, 218231. doi: 10.1016/j.bbabio.2011.05.017

Ptushenko, V. V., Cherepanov, D. A., Krishtalik, L. I., and Semenov, A. Y. (2008). Semi-continuum electrostatic calculations of redox potentials in photosystem I. Photosynth. Res. 97:55. doi: 10.1007/s11120-008-9309-y

Rappaport, F., Guergova-Kuras, M., Nixon, P. J., Diner, B. A., and Lavergne, J. (2002). Kinetics and Pathways of Charge Recombination in Photosystem II. Biochemistry 41, 8518-8527. doi: 10.1021/bi025725p

Rutherford, A. W., Osyczka, A., and Rappaport, F. (2012). Back-reactions, shortcircuits, leaks and other energy wasteful reactions in biological electron transfer: redox tuning to survive life in O2. FEBS Lett. 586, 603-616. doi: 10.1016/j. febslet.2011.12.039

Schoepp-Cothenet, B., Lieutaud, C., Baymann, F., Verméglio, A., Friedrich, T., Kramer, D. M., et al. (2009). Menaquinone as pool quinone in a purple bacterium. PNAS 106, 8549-8554. doi: 10.1073/pnas.081317 3106

Shinkarev, V. P., Vassiliev, I. R., and Golbeck, J. H. (2000). A kinetic assessment of the sequence of electron transfer from $F(X)$ to $F(A)$ and further to $F(B)$ in photosystem I: the value of the equilibrium constant between $F(X)$ and $F(A)$. Biophys. J. 78, 363-372. doi: 10.1016/S0006-3495(00)76599-4

Šnyrychová, I., Pospíšil, P., and Nauš, J. (2006). Reaction pathways involved in the production of hydroxyl radicals in thylakoid membrane: EPR spin-trapping study. Photochem. Photobiol. Sci. 5, 472-476. doi: 10.1039/B514394B

Takahashi, M., and Asada, K. (1988). Superoxide production in aprotic interior of chloroplast thylakoids. Arch. Biochem. Biophys. 267, 714-722. doi: 10.1016/ 0003-9861(88)90080-X

Taylor, R. M., Sallans, L., Frankel, L. K., and Bricker, T. M. (2018). Natively oxidized amino acid residues in the spinach cytochrome b6f complex. Photosynth. Res. 137, 141-151. doi: 10.1007/s11120-018-0485-0 
Turrens, J. F., Alexandre, A., and Lehninger, A. L. (1985). Ubisemiquinone is the electron donor for superoxide formation by complex III of heart mitochondria. Arch. Biochem. Biophys. 237, 408-414. doi: 10.1016/0003-9861(85)90 293-0

Vetoshkina, D. V., Ivanov, B. N., Khorobrykh, S. A., Proskuryakov, I. I, and Borisova-Mubarakshina, M. M. (2017). Involvement of the chloroplast plastoquinone pool in the Mehler reaction. Physiol. Plant. 161, 45-55. doi: $10.1111 /$ ppl.12560

Wardman, P. (1990). Bioreductive activation of quinones: redox properties and Thiol reactivity. Free Radic. Res. Commun. 8, 219-229. doi: 10.3109/ 10715769009053355
Conflict of Interest: The authors declare that the research was conducted in the absence of any commercial or financial relationships that could be construed as a potential conflict of interest.

Copyright (๑) 2020 Kozuleva, Ivanov, Vetoshkina and Borisova-Mubarakshina. This is an open-access article distributed under the terms of the Creative Commons Attribution License (CC BY). The use, distribution or reproduction in other forums is permitted, provided the original author(s) and the copyright owner(s) are credited and that the original publication in this journal is cited, in accordance with accepted academic practice. No use, distribution or reproduction is permitted which does not comply with these terms. 